\title{
J-NSE-Phoenix, a neutron spin- echo spectrometer with optimized superconducting precession coils at the MLZ in Garching
}

Cite as: Rev. Sci. Instrum. 90, 043107 (2019); https://doi.org/10.1063/1.5084303

Submitted: 06 December 2018. Accepted: 15 March 2019. Published Online: 05 April 2019

S. Pasini (D), O. Holderer (D), T. Kozielewski (D), D. Richter (D), and M. Monkenbusch (D)

CrossMark

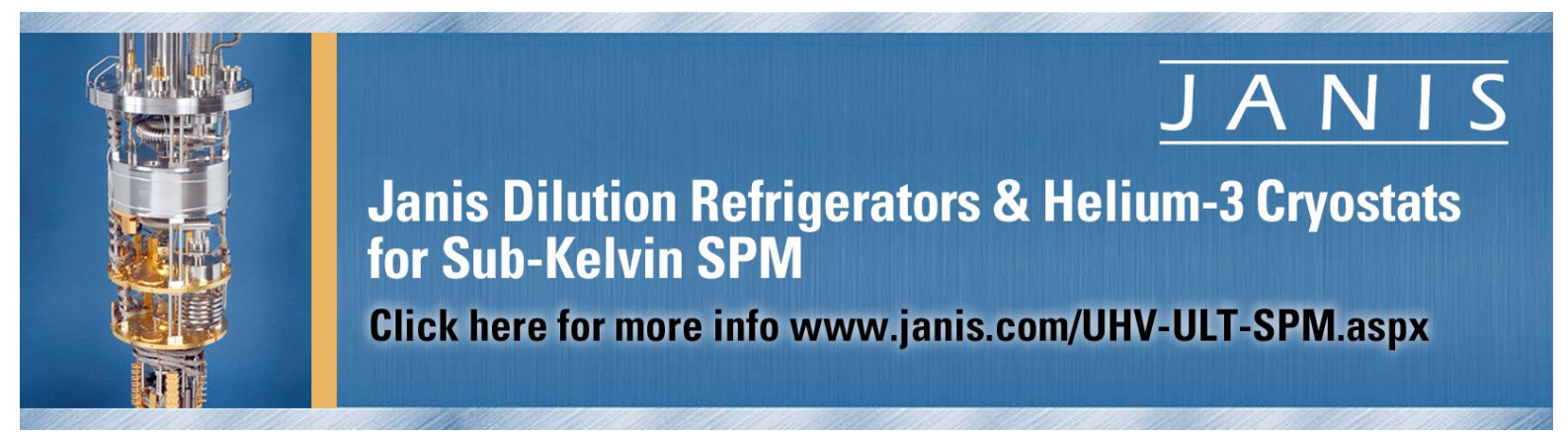




\title{
J-NSE-Phoenix, a neutron spin-echo spectrometer with optimized superconducting precession coils at the MLZ in Garching
}

\author{
Cite as: Rev. Sci. Instrum. 90, 043107 (2019); doi: 10.1063/1.5084303 \\ Submitted: 6 December 2018 • Accepted: 15 March 2019 • \\ Published Online: 5 April 2019
}

\author{
S. Pasini, ${ }^{1, a)}$ (D) O. Holderer, ${ }^{1}$ (D) T. Kozielewski, ${ }^{2}$ (D) D. Richter, ${ }^{3}$ (D) and M. Monkenbusch ${ }^{3}$
}

\begin{abstract}
AFFILIATIONS
${ }^{1}$ Forschungszentrum Jülich GmbH, JCNS at Heinz Maier-Leibnitz Zentrum, Lichtenberstraße 1, 85747 Garching, Germany

${ }^{2}$ Jülich Centre for Neutron Science (JCNS), Forschungszentrum Jülich GmbH, Jülich, Germany

${ }^{3}$ Jülich Centre for Neutron Science (JCNS) and Institute for Complex Systems (ICS), Forschungszentrum Jülich GmbH, Jülich, Germany
\end{abstract}

\begin{abstract}
a) Author to whom correspondence should be addressed: s.pasini@fz-juelich.de
ABSTRACT

A novel set of superconducting main precession coils has been built and installed in the Jülich-neutron spin-echo (J-NSE) spectrometer at the Heinz Maier-Leibnitz Zentrum (MLZ) in Garching. These unique new coils comprise a field-integral optimizing field shape, fringe field compensation, and high stability. They yield an enhancement of a factor of 2.5 in the intrinsic field-integral homogeneity, i.e., the resolution. The coil concept has been developed for the ESSENSE instrument proposal for the European Spallation Source. We report on the construction of and on the first results from the new superconducting neutron spin-echo spectrometer at the MLZ in Garching where the coils are the main part of a refurbishment of the J-NSE spectrometer after twenty years of operation.
\end{abstract}

Published under license by AIP Publishing. https://doi.org/10.1063/1.5084303

\section{INTRODUCTION}

Novel superconducting solenoid sets, replacing the previous precession coils of the J-NSE spectrometer in Garching, increase the useful resolution of the instrument by a factor of 2.5. The new coils have been designed following a field-integral homogeneity optimization goal for maximum resolution. Further simultaneously observed conditions were very low fringe fields, practical feasibility in the superconducting form, compatibility of the overall field shape with the functional requirements of the flippers and other genuine NSE elements, and finally also to fit into the available space as defined by the previous installation. ${ }^{2}$ The total development of this concept was mainly triggered and enabled by the preparation of the ESSENSE instrument proposal for the European Spallation Source (ESS). The present coil configuration is just a slight modification (enforced by the present length limitations) of the proposed ESSENSE design.

Spin-echo instruments are essential to extend the effective energy resolution of thermal neutron spectroscopy down to the $\mathrm{neV}$ range. This is possible because the spin precession of neutrons is used to encode tiny velocity changes, introduced upon scattering at a sample, into changes of the final spin state. ${ }^{4}$ As a result of the instrument's spin manipulations, the final polarization of the detected neutron intensity corresponds to the normalized intermediate scattering function

$$
I(Q, t) / I(Q, t=0)=\frac{1}{S(Q)} \int S(Q, \omega) \exp (i \omega t) d \omega,
$$

with $Q$ being the scattering wavevector and $S(Q, \omega)$ being the scattering function and $\omega$ relating to the energy transfer $\Delta E=\hbar \omega$. The spin evolution in the precession field region separated by the $\pi / 2$ flippers that define start and end and the "time reverting" $\pi$-flipper is illustrated in Fig. 1. At symmetry between the primary $\left(\pi / 2_{1} \rightarrow\right.$ $\pi$-flipper) and secondary ( $\pi \rightarrow \pi / 2_{2}$-flipper) neutron flight paths, the spin-precession angles cancel independent of the initial neutron velocity. Thus, the final polarization is the same as that at start. If inelastic scattering at the sample, which is located close to the $\pi$-flipper, changes the neutron energy, cf. its velocity, the final precession angle deviates from zero and the polarization is changed. Integration over many neutrons, the sampling velocity changes 


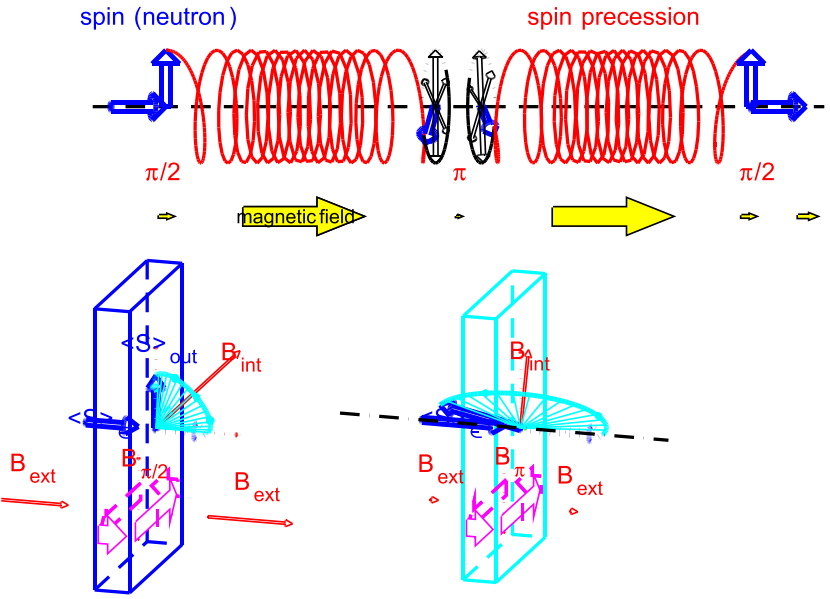

FIG. 1. Schematic setup of NSE and of its functional principals: The Larmor precession of the neutron spin between the $\pi / 2_{1,2}$ flippers and the central $\pi$ flipper, where the spin is rotated by $180^{\circ}$ (above). Below: the main components of the magnetic fields inside a $\pi / 2$ flipper.

according to the spectrum of the inelastic scattering leading to Eq. (1). The sensitivity of velocity changes depends on the magnetic field in the precession region; this determines the time parameter in Eq. (1) $t=J \lambda^{3} \gamma m_{n}^{2} /\left(2 \pi h^{2}\right)$, with $J=\int|B| d l$ being the field integral over the neutron path in one spectrometer arm, $\lambda$ being the neutron wavelength, $\gamma=2 \pi \times 2.91306598 \times 10^{7} \mathrm{~s}^{-1} / \mathrm{T}, m_{n}$ being the neutron mass, and $h$ being Planck's constant. Neutron spin-echo (NSE) spectroscopy is a Fourier method. The notion "resolution" for NSE instrument is commonly understood as the maximum value of $t$ for which useful experimental data can be collected. Typically, this is limited by the variance $\delta=\sqrt{\left\langle(\Delta J / J)^{2}\right\rangle}$ of the precession coding field integral $J$ values of neutron paths within the beam. In a coarse approximation, this leads to a reduction in the echo signal by a factor $\mathcal{R} \simeq \exp \left(-[\delta J g \lambda]^{2}\right)$ with $g=\gamma m_{n} / h$. In order to be useful, the resolution factor must be larger than $\mathcal{R} \simeq 1 / e$.

The new precession coils have been designed according to an optimization procedure in order to minimize the variation of magnetic field integrals along the paths within the neutron beam. ${ }^{2}$ The idea of this type of field shaping was originally investigated by Zeyen, ${ }^{5}$ and the spin-echo instrument iNSE (C2-2) ${ }^{6}$ at the JRR-3 reactor in Tokai was built according to the original scheme. More recently, this idea was extended to instruments with a divergent beam that comprises a larger solid angle, at least at the detector side. The 2016 refurbished solenoids of the ILL instrument IN15 were constructed accordingly. ${ }^{7}$ Furthermore, the viability of using dipole moment compensated superconducting coils as precession solenoids in a high resolution NSE instrument was demonstrated by the SNS-NSE at the Spallation Neutron Source (SNS) in Oak Ridge.

After 10 years of operation at the research reactor FRJ-2 in Jülich ${ }^{9}$ and 10 further years at the FRMII reactor in Garching, the original water-cooled copper precession solenoids of the J-NSE spectrometer were now (2017) replaced by the more sophisticated superconducting solenoid arrangements described here. The novel solenoid design achieves a reduction in the initial field integral inhomogeneity by a factor of 2.5 compared to previous generation designs. This reduction leads to a corresponding diminishment in the final $\delta$-value after correction and thus to a more than twofold extension of the Fourier time range at a given neutron wavelength $\lambda$. The previous coil design of the J-NSE spectrometer allowed to use a maximum field integral of $J=0.48 \mathrm{Tm}$ at lower wavelength, while at longer wavelength rather $\delta J$ was the limiting factor. At a wavelength of $\lambda=8 \AA$, the maximum useful Fourier time was between 35 and $40 \mathrm{~ns}$, and with the new coils, now it surpasses $90 \mathrm{~ns}$. Due to the superconducting coil-design, the new "J-NSE Phoenix" has important further advantages, besides the reduced power needs: (i) an efficient fringe field compensation that allows for easy operation at larger scattering angles due to minimal cross talk between the precession coils on both arms and (ii) decoupling of any position fluctuation (e.g., by thermal expansion of the main solenoids) from variations of the effective field integral $J$. The latter is a key to signal stability.

\section{INSTRUMENT LAYOUT}

\section{A. General}

The basic instrument layout had to be compatible with the geometry of the (old) J-NSE instrument. ${ }^{1}$ Thus, the length of the spectrometer arms and the positions of flippers and auxiliary solenoids had to stay at their previous values within a few centimeters. The mechanical supports and the bulk of auxiliary coils were also kept the same. However, the diameter of the main solenoid coils is increased, the outer dimensions of the cryostats just being compatible with the given support structure and beam height. A picture of the J-NSE setup with the new coils in place is shown in Fig. 2.

All auxiliary current sources were replaced by modern state-ofthe-art switching power supplies with full digital regulation loops. ${ }^{10}$

\section{B. Main precession coils}

The replacement of the previous water-cooled copper coils by the new optimized shape superconducting main precession coil sets was the core of the refurbishment of the J-NSE-spectrometer (Table I).

The new coil sets consist of 3 main sections and 2 axial fringe field modulators, each having an inner and an outer part (see the inset in Fig. 4). The outer coil parts serve to compensate the net dipole moment of the assembly; they basically "catch" the flux lines and return them in the space between inner and outer partial coils. The optimization computations revealed that increasing the number of partial coils beyond these 5 compensated sets yields, if any, only marginal improvement of the field integral homogeneity. ${ }^{2}$ Even the thus obtained 10 partial coils within one cryostat are a manufacturing challenge. ${ }^{11}$

All 10 partial coils of one arm are wound on an assembly of concentric rigidly connected aluminum cylinders that are kept at an operation temperature of 5-6 K inside a cryostat. This ensures that the geometry of the current carrying wire is absolutely stable. Any movement of the rigid assembly as a whole has a negligible influence on the field integral on paths between the flippers since the field of the rigid coil assembly is virtually zero at the flipper positions.

This is one of the important virtues of using superconducting coils. Furthermore, the geometrical accuracy of the effective current 


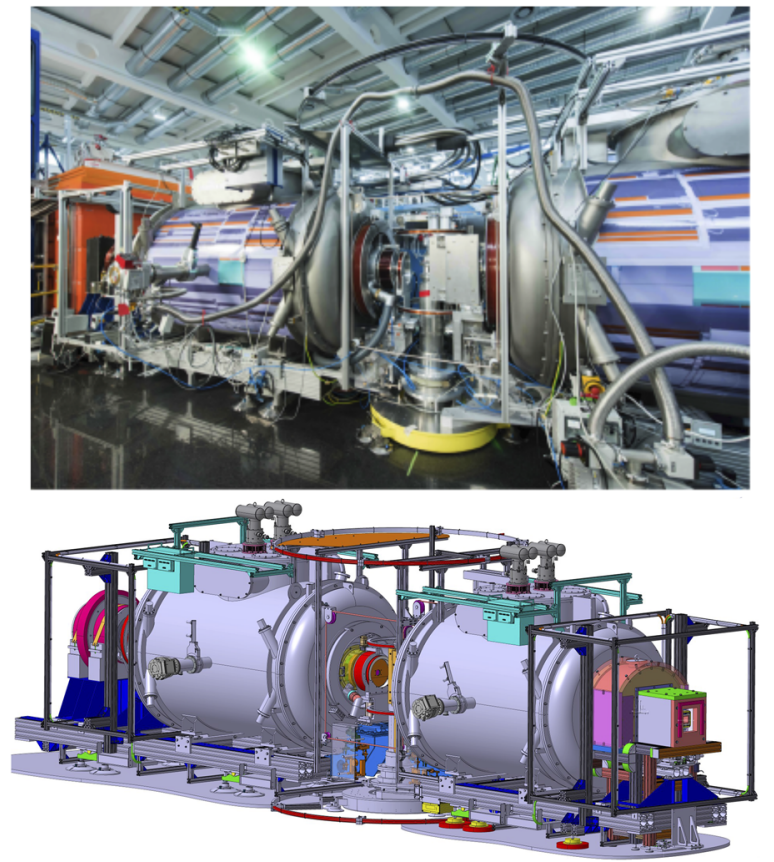

FIG. 2. Photo (image credit: Tobias Hase) and simplified sketch of the J-NSE Phoenix installation at the FRMII in Garching; most prominently shown are the two large cryostats that hold the main precession coil sets on the first (right) and second (left) spectrometer arms. The leftmost auxiliary coil shown in red in the sketch holds the analyzer mirrors, and the detector placed behind it is not shown here. The rightmost box-like structure on the other side is a lead shield around the beam entrance with a monitor counter and first $\pi / 2$-flipper. The secondary (left side) arm can be rotated around the sample position on air pads in order to set nominal scattering angles between $-1^{\circ}\left(3^{\circ}\right.$ for scattering) and $85^{\circ}$.

flow paths is-due to the at least 15 times smaller wire diametermuch better than in the case of the normal conducting coils. Moreover, the complete fringe field compensation (i.e., zero net dipole moment) for a coil with a comparable field integral is hardly possible to realize with normal conductors. In any case, the resulting assembly would be prohibitively massive and power consuming, e.g., even without complete compensation, the new normal conducting coils of the IN15 instrument at ILL dissipate about $700 \mathrm{KW}$ at $J_{0}$ $=1 \mathrm{Tm}$. The higher direct costs of the superconducting coil sets are largely compensated by the considerably less effort to install the electrical power and the corresponding cooling for an equivalent normal conducting coil. In addition, there will be significant savings of operation cost for electrical power and cooling water. The latter, however, depends somehow on the average modes of usage since the

TABLE I. Coil comparison.

\begin{tabular}{llcccc}
\hline \hline Coils & $\begin{array}{c}J_{\max } \\
(\mathrm{Tm})\end{array}$ & $\sqrt{\delta J^{2}} / J_{0}$ & $\begin{array}{c}\text { Current } \\
(\mathrm{A})\end{array}$ & $\begin{array}{c}\text { Wire size } \\
(\mathrm{mm})\end{array}$ & $\begin{array}{c}\text { Power } \\
(\mathrm{KW})\end{array}$ \\
\hline $\mathrm{Cu}$ (old) & 0.48 & 900 & 440 & 14 & 200 \\
$\mathrm{NbTi}($ new $)$ & 1.5 & 350 & 220 & 0.9 & 32 \\
\hline \hline
\end{tabular}

cooling power for the superconducting coils has to be supplied continuously, irrespective of spectrometer settings, whereas the normal conducting coil power directly depends on settings. A reasonable estimate is $1 / 3$ of the maximum power during neutron production cycles. In numbers (assuming $700 \mathrm{KW}$ maximum power), this would be $32 \times 24 \times 365=280.32 \mathrm{MWh} /$ year for the superconducting system compared to (assuming between 120 and 200 day neutron operation per year) between $700 / 3 \times 24 \times 120=672 \mathrm{MWh}$ and $1120 \mathrm{MWh}$ for a normal conducting system. But we emphasize that this is just an additional benefit that comes with the essential features of fringe field compensation and geometric winding accuracy and stability.

The layout of the new J-NSE Phoenix superconducting coil set is illustrated in Fig. 3. Figure 4 displays the field along the neutron path through the solenoids together with the arrangement of the active windings (top inset). The curve is not as smooth as the ideal theoretical one from the semi-analytical optimization in Ref. 2 but, nevertheless, fulfills all conditions concerning low intrinsic field integral inhomogeneity, compatibility with a correction scheme with two parabolic correctors only, and efficient fringe field compensation. The knobby shape at the maximum is a consequence of the restriction to the lowest possible number of simple cylindrical partial coils that still meet the abovementioned conditions.

\section{Hysteresis effects}

A particular concern connected with the use of superconducting coils in a spin-echo spectrometer pertains the hysteresis fields due to trapped flux in the type II superconducting wires. However, the hysteresis influence on NSE performance is small and benign since:

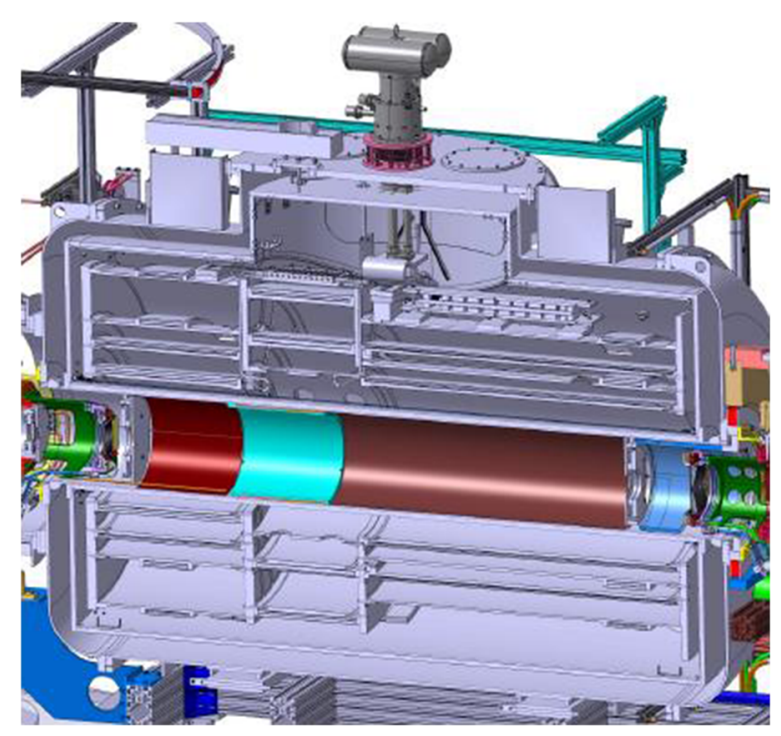

FIG. 3. Cut through one main solenoid assembly giving a view inside the coils and the warm bore installations. The light green cylinder in the warm bore indicates the phase coil, and the dark red indicates a lining with boron absorber. At both ends, held by the green cylindrical supports the correction elements can be seen. Further elements in the warm bore are current sheets (shifters) and circular boron absorber apertures. 


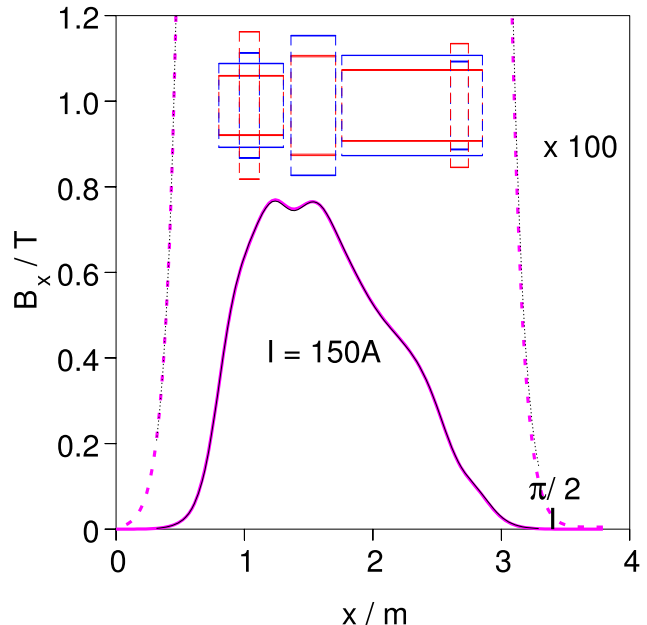

FIG. 4. Field on the axis for a current of $150 \mathrm{~A}$ for the coil sets in cryostat $\mathrm{A}$ (one spectrometer arm) comparing measurement (thick magenta line) and model calculation with slight fitted adjustment of the geometric parameters compared to those underlying the construction (thin black line on top of the thick line). The fit curves and the measurement coincide within the plot line-width. The upper part shows a cut through underlying superconducting coil conformation (only showing the active current conductor winding). The arrangement consists of 5 compensating pairs (red-blue), with minor exceptions: inner windings consist of 8 layers, and outer windings consist of 4 layers (shorter large ring coils have 2 or 4 extra layers). For manufacturing reasons, spaces between the segment coils are needed, so they have been included during the whole optimization of this geometry.

- The volume occupied by the superconductor is small. The thicknesses of the coils are $8 \mathrm{~mm}$ for the inner and $4 \mathrm{~mm}$ for the outer windings as compared to the diameters that all exceed $450 \mathrm{~mm}$; moreover, the used wire has a diameter of $0.825 \mathrm{~mm}$ plus isolation, the same type as was used for the coils of the SNS-NSE spectrometer. It has a comparatively low hysteresis; see the inset in Fig. 5 and Ref. 8. Nevertheless, as shown in Fig. 5, we observe residual fields in the $10^{-4} \mathrm{~T}$ range after a cycle up to $150 \mathrm{~A}$ and return to $0 \mathrm{~A}$.

- The physical requirement is that the net circulating current in the winding must be zero; when the external current is forced to zero, Amperes law $\int_{-\infty}^{\infty} \vec{B} \cdot \overrightarrow{d l}=\mu_{0} I$ (with $I$ being the total current circulating around the path along $\overrightarrow{d l}$ ) implies that for a sufficient length of the considered path, the field integral effect is virtually zero. For off axis paths used typically in an NSE-experiment, this is still approximately valid. The computed absolute effect (maximum at lowest field) of the hysteresis fields on the phase over a path limited by the flipper positions corresponds to $\Delta J \simeq 4.3$ $\times 10^{-5} \mathrm{Tm}$, about $20 \%$ of the effect of typical ambient fields ("earth field") in a guide hall. Both influences are tolerable if they are constant within a few tenth of a percent.

To compensate for this potential phase deviation, the two solenoid sets are as identical as possible, having the same geometry and corresponding partial coils being wound from the same wire batches such that any residual hysteresis effects will cancel as long as both coils exhibit the same current history. The latter is

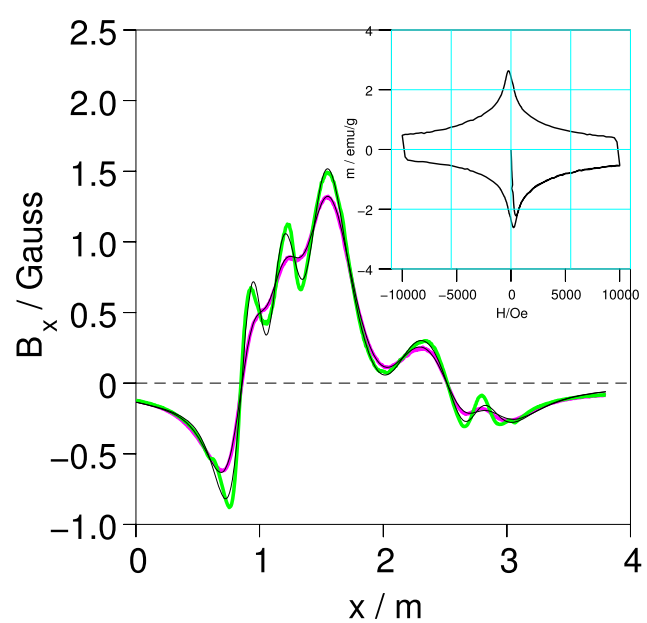

FIG. 5. Residual hysteresis field on axis (thick magenta line) and $125 \mathrm{~mm}$ parallel to it (thick green line) after a current cycle of 0 A-150 A-0 A. The measured curves are displayed together with the result of a simplified empirical model (black thin lines), confining current to the winding volumes and zero integral circulating current. To empirically model the hysteresis curve, the windings in each coil are considered as two counteracting layers that fill the original volume of the winding. The total circulating current must be zero. The virtual layer currents of the resulting model are fitted such that the computed field matches the measured curve. The inset shows the (measured) hysteresis curve for the used wire type with the field direction perpendicular to the wire axis.

ensured by operating both coil sets from the same current source in series.

The simple approximate model for the hysteresis field that reasonably fits the measured residual field in Fig. 5 was also used to assess the possible influence on the field integral homogeneity. The results show that this influence is far below from being an issue; see Sec. IV for experimental verifications.

\section{CONFIGURATION OF THE SECONDARY SPECTROMETER}

An overview of the spectrometer configuration in terms of functional magnetic elements is shown in Fig. 6. The set of 10 cylindrical coils in the center of each arm represents the superconducting partial windings; supports and cryostats are only shown in the lower part. The large current rings around the central sample position as well as the large rectangular/square type coils consisting of 1 or two windings that surround the flipper locations serve to compensate environmental fields there. The somehow smaller concentric coaxial coils tailor the longitudinal fields needed by flippers and serve to adiabatically transport the neutron spins. At the outer sides (right), a coil to turn the polarizer field and (left) a coil set containing the analyzer mirrors are shown.

The field in the sample region is first of all provided by two $0.8 \mathrm{~m}$ diameter ring coils at $0.6 \mathrm{~m}$ distance from the sample, each attached to the main coil cryostat on the primary or secondary spectrometer arm. These enable precise control of the longitudinal field component (i.e., perpendicular to $\vec{Q}$ ) at the sample. Further smaller ring coils (solpic) between the $\pi$-flipper and main coil serve to reduce the residual fringe field of the main coil at the flipper position. 

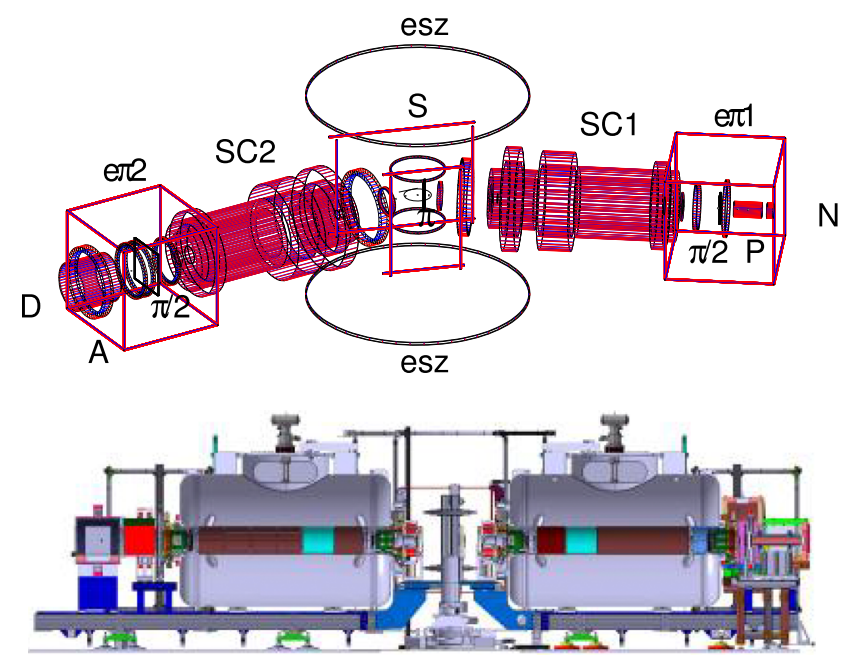

FIG. 6. Spectrometer solenoid configuration shown for $Q=0.5 \AA^{-1}$ at $\lambda=8 \AA$ (upper part). Only active current carrying elements are shown. $S$ indicates the sample position, $D$ indicates the detector position, $A$ indicates the analyzer, $P$ indicates the polarizer, and N indicates the entry point of the neutron beam. SC1 and SC2 are the superconducting main solenoid sets (cryostats not shown). Flippers are indicated by $\pi / 2$ or $\pi$. $e \pi 1$ and $e \pi 2$ are one turn rectangular coils to compensate the "earth field" components at the $\pi / 2$-flipper positions and esz indicates vertical compensators for the sample region (2 turns). Short spin-echo and phasecoils are omitted for clarity. The lower part shows a side view of the spectrometer construction including the mechanical supports and cryostats.

A pair of $0.6 \mathrm{~m}$ diameter ring coils concentric to the sample table column at $0.3 \mathrm{~m}$ above and below the scattering plane allows to set the $\mathrm{z}$-component of the magnetic field embedding the $\pi$-flipper to a (small) value that ensures proper flipper operation by rendering the field direction outside of the flipper perpendicular to that inside the flipper. Larger two turn ring coils (diameter $2.4 \mathrm{~m}$ ) at the floor and $1.2 \mathrm{~m}$ above the sample can be used to compensate the vertical earth field component. A shape-variable one-turn coil that adapts to the scattering angle dependent geometry changes allows us to create an extra field component in the scattering plane that is perpendicular to the longitudinal component (i.e., parallel to $\vec{Q}$ ). In case of magnetic scattering, these coils can be used to orient the magnetic-field vector at the sample position either parallel or perpendicular to the scattering vector, for a full-polarization analysis.

On the other sides of the main solenoids, several ring coils with diameters around $0.6 \mathrm{~m}$ tailor the longitudinal field environment of the $\pi / 2$-flippers and the transport from polarizer and to the analyzer sections. The first of these coils is attached to the outer hull of the cryostats and effectively reduces the residual fringe field at the flipper positions. Together with ring coils on the outer sides of the $\pi / 2$-flippers, they are controlled such that the necessary longitudinal embedding field for the flippers is generated (typically several Gauss). The $\pi / 2$-flipper zones are surrounded by one turn square shaped current loops that compensate for external earth field components. The ring coils that are symmetric in both arms are electrically in series. Their magnetic contribution typically corresponds to values between 100 and 1000 A turns.

Finally at the detector side, a solenoid with an outer compensation surrounds the analyzer to sustain the magnetization of the magnetic multilayers. At the polarizer side, a short solenoid around the neutron path between the polarizer and spectrometer entrance adiabatically turns the polarization direction from the transversal polarizer direction to longitudinal.

Inside the warm bore of the cryostats, cylindrical coils with $2 \times 80$ windings are inserted in each side. A suitable selection or combination of these serves a symmetry coil and is scanned during the elementary "phase scans."

Furthermore, the important correction elements are mounted at the entrance and exit of the cryostats warm bores, as can be seen in Fig. 7. They can be accurately positioned in the plane perpendicular to the beam axis by amagnetic piezo drives.

In the sample area, the room between the two "solpic" coils amounts to circa $700 \mathrm{~mm}$. In the middle of this area, the sample column is located. The height of the sample platform can be controlled by a motorized lift with a travel of circa $200 \mathrm{~mm}$. On the one side of the sample column, $140 \mathrm{~mm}$ from its center, the $\pi$-flipper is located, allowing for a maximum diameter of the sample environment of $250 \mathrm{~mm}$ in a standard configuration.

The detector is a Denex ${ }^{3} \mathrm{He} 32 \times 321 \mathrm{~cm}^{2}$ multidetector, positioned $4.4 \mathrm{~m}$ from the sample.

Extra coils and flippers to extend operation to 10 times lower field integrals (i.e., short Fourier times) are installed in the sample region. The short-Fourier-time setup is mainly a heritage from the previous installation. This mode allows us to extend the minimum useful Fourier time range limit by about one order of magnitude. All precessions in this mode are performed in the sample zone that the neutrons enter after the beam left (or prior reentering) the main solenoid sets. Here the main precession coils only serve as guide fields. An extra pair of $\pi / 2$-flippers limits the precession, and the field integral is controlled by short and compensated extra solenoids

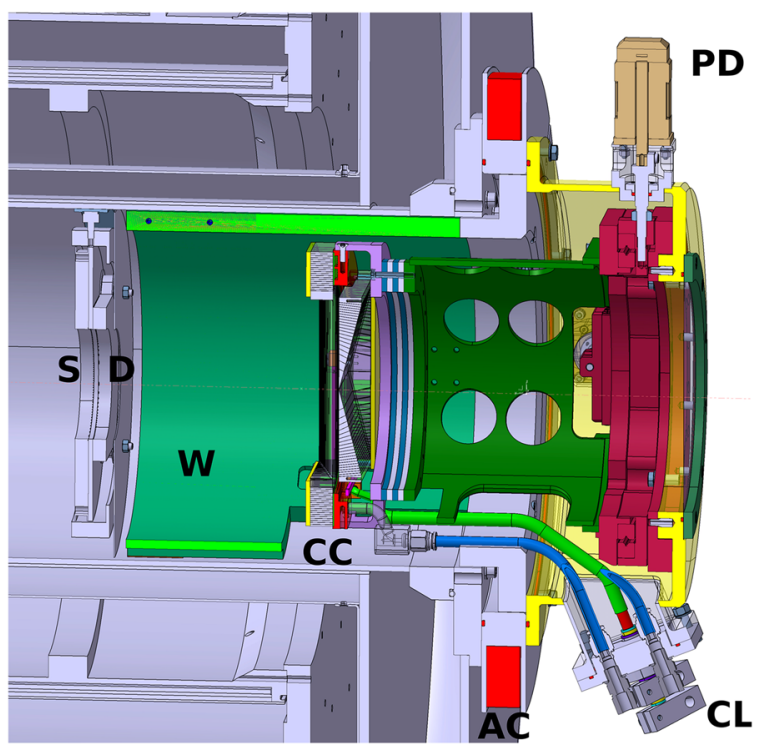

FIG. 7. Mounting of a correction element at the $\pi / 2$-flipper side of the main coil. With S: shifters, D: diaphragm, W: tungsten insert as $\gamma$-shield, CC: correction coil assembly, CL: connectors for CC-current and cooling water, PD: piezo drive, and AC: (integrated) auxiliary ring coil. 

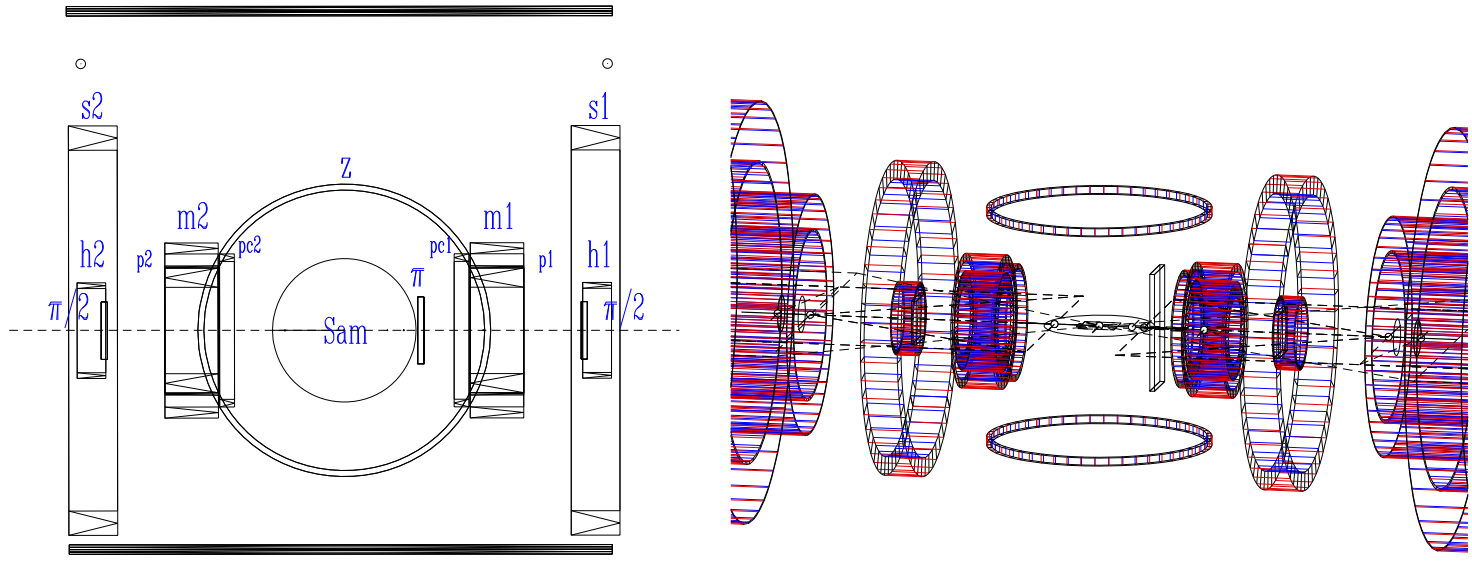

FIG. 8. Active elements in the short time NSE setup. s1, s2: sample field coils on the outside of the cryostats (also used in normal operation). h1, h2: auxiliary coils to keep the longitudinal $\pi / 2$-flipper at the required values. $\mathrm{m} 1, \mathrm{~m} 2$ : compensated main short-time precession coils; these also carry the phase symmetry coils: $\mathrm{p} 1, \mathrm{p} 2$, which are operated in antiparallel mode. pc1, pc2: compensation for the $\pi$-flipper field (optional use). $z$ : the standard $z$-coils to set the $z$-component of the embedding field for the $\pi$-flipper. The main solenoid (parts of it are still displayed in the 3D view at the right side) serves as a guide field only and is operated at a fixed low current.

(m1, $\mathrm{m} 2$ in Fig. 8). With that, the effective field integral can be reduced down to $10^{-4} \mathrm{Tm}$. More details on the magnetic field configuration can be found in the supplementary material.

\section{A. Correction elements}

Even field integral homogeneity optimized main coils need correction elements to further reduce the residual inhomogeneity by at least two orders of magnitude. Ideally, a perfect correction would be achieved by radially symmetric current density distributions at three positions along the beam axis and each covering the neutron beam cross section area. ${ }^{4,13,14}$ The required radial current density increases (approximately) linearly with the distance from the magnetic axis. At the outer periphery, the needed maximum projected current density values are of the order of up to $50 \mathrm{~A} / \mathrm{mm}$. Thus, the physical realization of such elements must be able to carry the corresponding non-trivial current density and at the same time be transparent to the neutron beam.

The main solenoid optimization was performed such that closeto-optimal field integral homogeneity was achieved after correction with just two radial current distributions with a linear radial current density function $j_{1,2}(r) \propto r$ at optimized positions and minimum necessary strength. The effective central axial positions for the two correction coils are about $25 \mathrm{~mm}$ "inside" the active winding zone of the main solenoid set at the sample side (ccr1) and about $70 \mathrm{~mm}$ "outside" the last active winding of the superconducting winding.

Thus a computed, theoretical rms field integral homogeneity of about $1 \mathrm{ppm}$ over the scattered beam was predicted.

The actual correction elements are of the so-called "Pythagoras" type, which were already employed at $\mathrm{IN}_{15} 5^{7}$ and in the SNSNSE spectrometer and the previous version of the J-NSE, ${ }^{8}$ the latter design is also used here. However, the new optimization compared to the SNS-NSE allowed the omission of the 3rd (middle) correction element in each arm. Thereby, the transmission is improved and the role of residual errors in the correction elements is reduced. The "Pythagoras" type implies that the radial current density that affects a correction action $\Phi(r) \simeq \mu_{0} \int j(r) d r \propto r^{2}$ is replaced by two orthogonal linear current zones each with an effect $\Phi_{x, y}(x) \propto x^{2}$ or $\propto y^{2}$ with a combined effect $\Phi \propto x^{2}+y^{2}=r^{2}$, cf. the name.

Figure 9 shows a sketch of the assembly. The return currents (beneath the dark yellow cover plates) to close the circuit of the linear zones adds to further (unwanted) field contributions of each half of the Pythagoras coil sets, and further the $\mathrm{x}$ - and $\mathrm{y}$-parts must have a small $(5 \mathrm{~mm})$ axial separation from each other. Both properties lead to a slight deviation from the ideal parabolic correction function and contribute to the minimum obtainable homogeneity. A quantitative view to these effects is provided by Figs. 14 and 15 in Sec. IV B. The carrier ring (red) consists of copper and serves as heat sink; it is water cooled (not shown here).

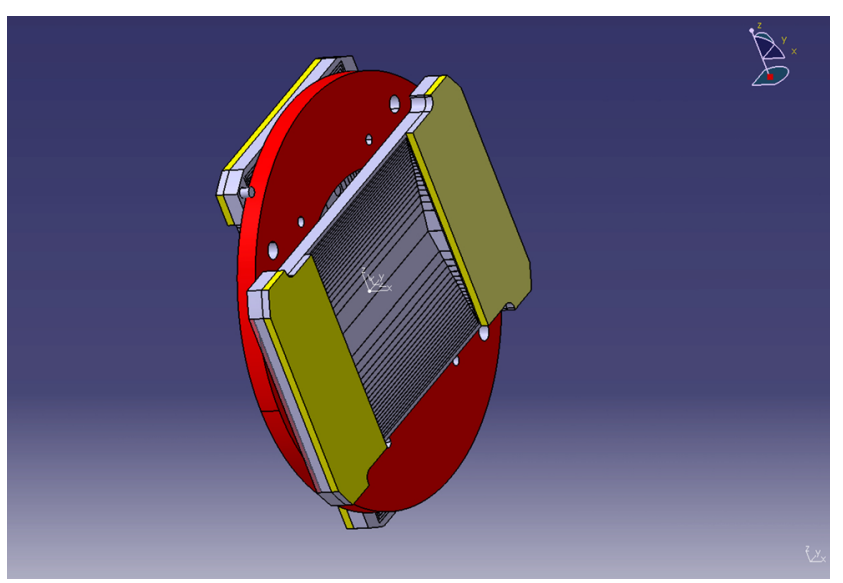

FIG. 9. Sketch of the correction coil for $100 \mathrm{~mm}$ beam width 
At the detector side (and for symmetry reasons also on the entering side of the neutron beam), larger coil sets of this type with $160 \mathrm{~mm}$ active width are installed. To reduce errors due to the cuts between zones, here the cuts are oblique such that the projected current density function is as smooth as possible.

We also simulated the effects of the real geometry of (Pythagoras) coils in the homogeneity computation. For this purpose, the field of the Pythagoras coils in use was tabulated with a hierarchy of 4 levels of spatial resolution covering the potential beam volume around the correction element and along the beam extension. The tables were created using the actual geometry of the construction drawings for the current paths. Using these tables, the program used for operation, assessment, and design of the J-NSE spectrometer yields the field integral values for any given operational state and set of paths. The peculiarity here is that the fieldintegrals have been calculated starting from a comprehensive "real" geometrical model of the whole spectrometer. A residual fieldintegral inhomogeneity of $2-3 \mathrm{ppm}$ at the center of the coils is to be expected.

Each correction element can be positioned perpendicular to the magnetic axis by two piezo drives ${ }^{15}$ to adjust their effective centers to the true magnetic axis. The whole system of actuators and amagnetic precision guide rails ${ }^{16}$ can easily cope with forces due to weight and magnetic forces up to $500 \mathrm{~N}$. The overall positioning accuracy with and without this load is better than $50 \mu \mathrm{m}$. In addition, current sheet type "shifters" are installed to allow fine tuning of the effective corrector centers to cope with scattering angle depended offsets due to residual magnetic cross talk between the spectrometer arms (see also Sec. IV D) and/or gravitation effects on slow neutrons at long wavelength without accumulating potential mechanical positioning hysteresis. The computed necessary shifts are less than $0.5 \mathrm{~mm}$ even for large scattering angles. The corresponding equivalent shifter current densities are well below $1 \mathrm{~A} / \mathrm{mm}$.

If the overwhelming source of the field integral inhomogeneity is the main solenoids, the currents in the main radial correctors must be proportional to the main current. This is the default setting. However, closer inspection of the phase maps reveals slight but distinct deviations. The linear coupling of CC-currents to the main current leads to phase map bulging at intermediate Fourier times associated with a minor drop of resolution. The effect recovers at maximum current since that was determined by optimizing the resolution using the neutron signal. The reason for the intermediate drop (i.e., nonlinearity) is the influence of (mainly) the solenoid that compensates the residual main solenoid fringe field at the $\pi / 2$-flipper position. It varies differently than the main current and adds to the inhomogeneity.

The nonlinearity has been computed using the field modeling built into the NSE operation program; however, with the current computing power and without parallelization (which might only be done with some effort), the processing is a factor 10-100 too slow to do the computation in real-time during a scan. Thus, the determination has been performed offline and a correction function was modeled to the results. It turns out that the main change pertains the current in the large correction coils. The computed results have been fitted to an empirically guessed function

$$
I_{\mathrm{CCRx}} \simeq \alpha I_{\text {main }}+\beta\left[1-\exp \left\{-\left(I_{\text {main }} / I_{x}\right)^{\gamma}\right\}\right]+\Delta \text {. }
$$

TABLE II. Typical computed CC3-current function coefficients for Eq. (2).

\begin{tabular}{lccccccc}
\hline \hline$\lambda / \AA$ & $Q / \AA^{-1}$ & $B_{\text {sample }} / \mathrm{G}$ & $\alpha$ & $\beta / \mathrm{A}$ & $\gamma$ & $I_{x} / \mathrm{A}$ & $\Delta / \mathrm{A}$ \\
\hline 6 & 0.1 & 1.6 & 0.38430 & -2.6075 & 0.498 & 35.298 & 0.149 \\
7 & 0.1 & 1.4 & 0.38449 & -2.5567 & 0.497 & 33.298 & 0.171 \\
8 & 0.1 & 1.4 & 0.38454 & -2.5694 & 0.498 & 31.689 & 0.201 \\
12 & 0.1 & 1.5 & 0.38383 & -2.6837 & 0.522 & 25.496 & 0.317 \\
\hline \hline
\end{tabular}

In the simple linear setting strategy, only $\alpha I_{\text {main }}$ with a slightly different $\alpha$-value is present. Typical values for all the nonlinearity parameters are given in Table II.

The correction according to Eq. (2) was incorporated into the NSE operation program and verified experimentally (see the supplementary material) to yield an optimal flat phase map for all $\tau$-settings.

\section{B. Flippers}

The flippers consist of rectangular coils wound with bare Alwire; the dimension perpendicular to the beam axis is $13 \mathrm{~mm}$. Their operation aspects are basically the same as in the previous version of the instrument; ${ }^{8,9}$ we just repeat a short summary of the main aspects. The full compensation of the main solenoids with virtually zero field on the axis at the flipper positions is an efficient measure to ensure this reliably. Thus, a rigid main solenoid configuration including compensation inside the cryostat at low constant temperature and on one contiguous winding support leads to virtually complete insensitivity of the phase (i.e., effective field integral) from moderate position fluctuations of the flipper or main solenoid positions.

The proper embedding fields needed for the intended flipper spin-rotations are then supplied by 2-3 extra ring coils (Helmholtz coils), each with a field contribution of a few Gauss only. The $\pi / 2$-flippers at the outer boundaries of the echo coding beam sections are operated such that the internal field component along the $\mathrm{z}$-axis equals the vertical field-component along the beam axis.

\section{OPERATION}

\section{A. Commencing}

The first echo was seen a month after installation at the instrument, and the final acceptance test was concluded. It was obtained for $1 \mathrm{~ns}$ at $8 \AA$ without extra corrections and the automatically computed current settings. This achievement is an elementary consistency and function test. It proves the proper symmetry of the spectrometer setup and the basic functioning of the flippers.

To operate the spectrometer at full resolution, the correction elements "Pythagoras-coils" must be employed and be properly adjusted. While the current settings can be predicted within $5 \%$, the exact positions with respect to the beam axis are a priori only known with an accuracy of $\simeq \pm 1-2 \mathrm{~mm}$ and must be adjusted using the neutron echo signals. The procedure is described in the supplementary material. Using this swiftly, a 50\% (average) and 77\% (center) resolution at $J_{0} \simeq 1 \mathrm{Tm}$ at $\lambda=8 \AA$ could be reached. 


\section{B. Measured properties: Hysteresis effects}

A basic early check pertains the verification of the computation result that the resolution is not affected by hysteresis fields by flux trapping in the superconductor.

The hysteresis field is largest at the lowest currents. Thus, the fact that the measured spin-echo signals at low Fourier time settings exhibit virtual full $(>0.95)$ resolution (see Figs. 10 and 11) immediately proves that there is no detectable hysteresis field related deterioration of resolution.

This observation is in accord with field integral computations using the model used also in Fig. 5 as an estimate for the extrahysteresis fields. No detectable extra field integral inhomogeneity is discernible.

Operation so far also gave no hint that the difference in the additional field integral contribution of the hysteresis field between both main coil sets gave rise to any observable symmetry phase point shifts during an experiment.

\section{Resolution and phase maps}

The ultimate resolution depends on the proper and accurate action of the correction elements. Conveniently one of them, the last one in front of the detector, allows for a position resolved inspection of its action in terms of phase shift, i.e., field integral deviation. The

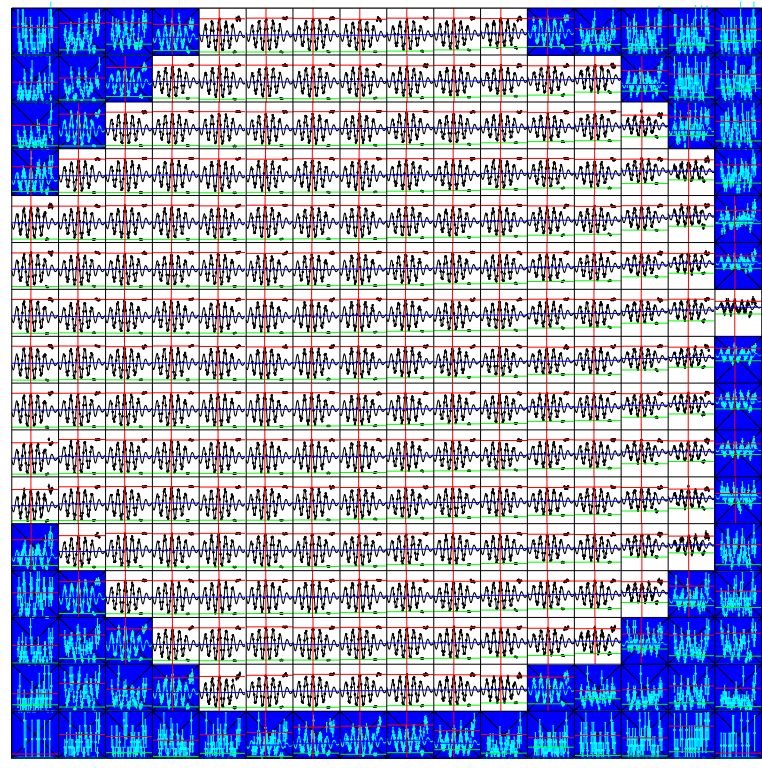

FIG. 10. Low Fourier time $\left(J=5.3 \times 10^{-3} \mathrm{Tm}, 0.5 \mathrm{~ns}\right.$ at $\left.\lambda=8 \AA\right)$ mapping of phase scan data to $2 \times 2 \mathrm{~cm}^{2}$ detector bins (grid boxes). The intensity variation results of the carbon powder resolution reference sample used here has been compensated by normalizing each partial scan to its maximum count. The circular footprint shape of beam intensity on the detector results from the apertures restricting the scattered beam in order to reduce background. Horizontal red, blue, and green lines through the in-cell echo signal plots indicate the "up," averaged, and "down" levels, and the vertical red lines indicate the symmetry phase locations. Note that the apparent flipping-ratio on the right side seems to be reduced. This is due to the significant drop of carbon powder scattering intensity toward this "larger"- $Q$ side of the detector causing general background contributions to become more important.

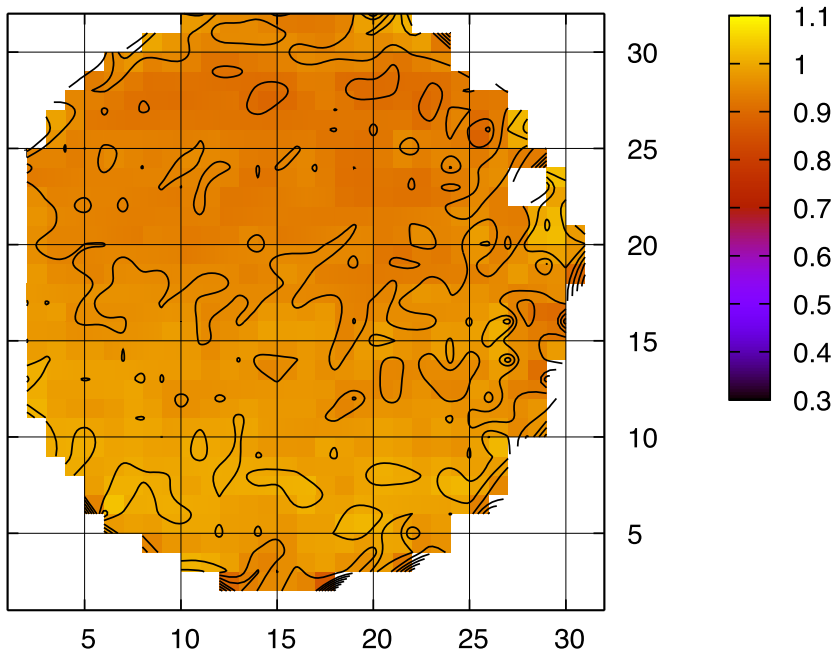

FIG. 11. Map of resolution at $\left(J=0.005 \mathrm{Tm}, 0.5 \mathrm{~ns}\right.$ at $\lambda=8 \AA$ ) and $Q=0.12 \AA^{-1}$ with correction elements in effect.

available spatial resolution is in the order of $1 \mathrm{~cm}$. Figures 12 and 13 show the resolution as a function of detector pixel position for a large field-integral/Fourier time (at the $50 \%$ average resolution limit, the pixels are weighed by their average intensity).

The nominal correction function is $\Phi(r) \propto r^{2}$, which is also by design the first order of the "Pythagoras-coil" assemblies. The next order of deviation from ideality, i.e., residual correction errors,

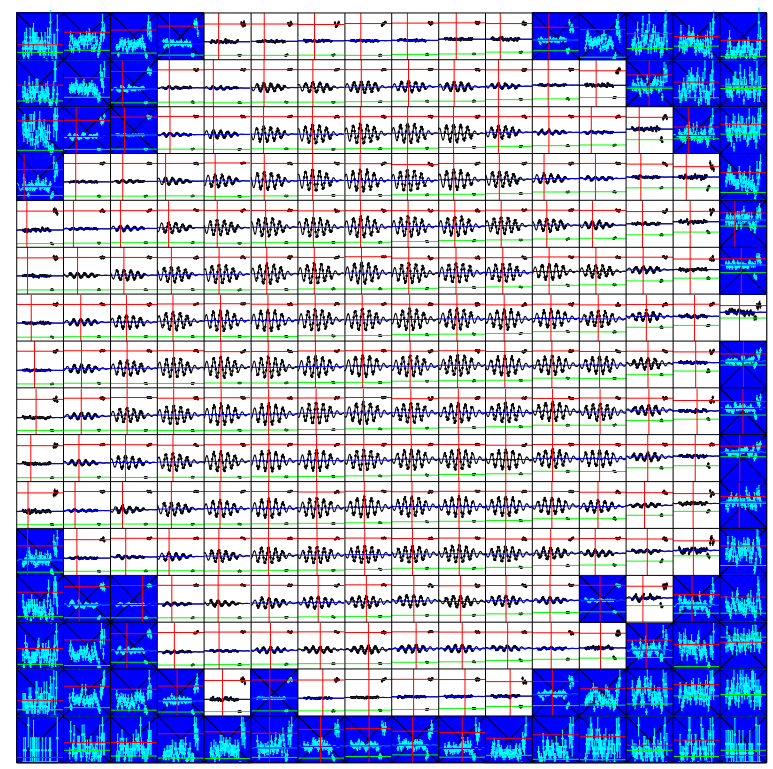

FIG. 12. Large Fourier time $(J=0.94 \mathrm{Tm}, 90 \mathrm{~ns}$ at $\lambda=8 \AA)$ mapping of phase scan data to detector bins, exhibiting the detector area over which the correction elements are effective. Dark blue crossed bins are rejected due to too weak signal contents. Note that the intensities are normalized to the maximum count in the respective "phase scan." 


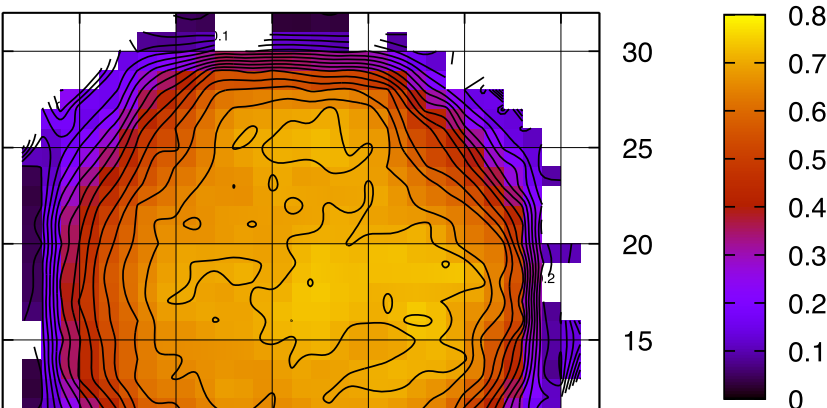

10

5

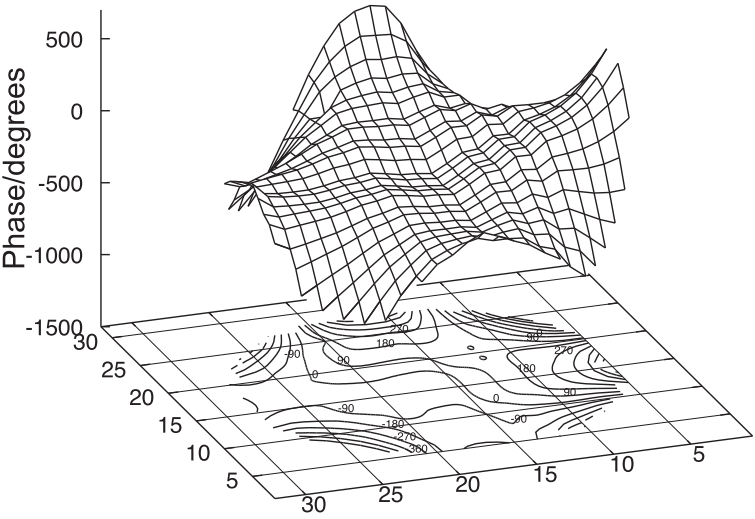

FIG. 15. Computed residual phase angle difference at $(J=0.94 \mathrm{Tm}, 90 \mathrm{~ns}$ at $\lambda$ $=8 \AA$ ), average over $2 \times 2$ points at the sample position $( \pm 0.0075 \mathrm{~m}, \pm 0.0075 \mathrm{~m})$.

plane perpendicular to the beam; see the supplementary material for more maps. The fact that the measured phase map coincides with computed map again shows that the large magnetic field from the superconducting coils matches the theoretical expectation without any detectable deviation.

A quick estimate of a phase-map variation on the resolution within a (small) pixel of size $\Delta r$ is given by

$$
\mathcal{R} \simeq \frac{2 \sin (\Delta r \operatorname{grad} \Phi / 2)}{\Delta r \operatorname{grad} \Phi} .
$$

Equation (3) can be obtained by averaging the cosine-function of the echo over a zero-phase $\Phi$ that varies linearly with $\delta r \times \operatorname{grad} \Phi$ over the pixel-size from $-\Delta r / 2-\Delta r / 2$. The phase accumulated by the neutron inside the precession area is proportional to the magnetic field-integral $J(x, y)$ and to the wavelength of the neutron, ${ }^{4} \Phi(x, y)$ $=\left[J(x, y)-J_{0}\right] \lambda \gamma m_{n} / h$, with $\gamma$ being the neutron Larmor constant, $m_{n}$ being the neutron mass, and $h$ being the Planck constant $\left(\gamma m_{n} / h\right.$ $=4.627 \times 10^{14} \mathrm{~T}^{-1} \mathrm{~m}^{-2}$ ).

Thus if the phase variation $\delta=\Delta r \operatorname{grad} \Phi$ over a pixel is less than $90^{\circ}$, the resolution stays above 0.9 . This means that concerning the last correction element in front of the detector, pixel-wise evaluation can significantly correct smooth variations of the phase map, as can be seen from Table III.

TABLE III. Resolution $\mathcal{R}$ as a function of detector pixel size for a setting of $\tau=90 \mathrm{~ns}$ at $\lambda=8 \AA\left(J_{0}=0.95 \mathrm{Tm}\right)$. The relative area is the fraction of pixels that is used to determine the average $\mathcal{R}$ for smaller pixel size; this represents approximately the aperture of the circular beam and illumination region at the detector.

\begin{tabular}{lccc}
\hline \hline Pixel size $(\mathrm{cm} \times \mathrm{cm})$ & $\mathcal{R}_{\max }$ & $\mathcal{R}_{\text {average }}$ & Relative area \\
\hline $32 \times 32$ & 0.049 & 0.049 & 1 \\
$16 \times 16$ & 0.316 & 0.167 & 1 \\
$8 \times 8$ & 0.580 & 0.241 & 0.94 \\
$4 \times 4$ & 0.706 & 0.355 & 0.83 \\
$2 \times 2$ & 0.724 & 0.445 & 0.74 \\
$1 \times 1$ & 0.770 & 0.495 & 0.72 \\
\hline \hline
\end{tabular}


However, for the other correction elements, the correlation between $(x, y)$ and the detector pixel is lost and the phase difference effects have to be integrated over the whole beam cross section at the positions of the respective elements. Note that unfortunately, the resolution effects as expressed by $\mathcal{R}$ of the correction elements residual errors are not multiplicative, but rather the phase angles accumulate and the total phase difference enters the cosine type integrands of the resolution expression, yielding a larger signal deterioration than a mere multiplication of individual $\mathcal{R}$ would give. The relevant cross sections in the incoming beam are of course significantly smaller than that on the detector side; thus, basically only the central plain parabolic zone of the correction is relevant. The residual deviations there determine the limit of the resolution at pixel size $\rightarrow 0$ at the detector.

Another possible source of inhomogeneity is deviations of current density in the beam region of the correction elements on a shorter length scale, e.g., gaps in the conductor, possible resistance fluctuations over the conductor zone by the spatial material, or temperature variation. These effects cannot be resolved in the measured phase-maps; their effect would be a general reduction in the observed resolution, even in the single pixels.

With the characterization experiments for the instrument that could be performed until now, we are able to extract an exact image of the centimeter scale of the action of the last large Pythagoras coil and corroborate the computation based on the real conductor geometry of the construction. However, in order to match the current through the correction coil (setting $90 \mathrm{~ns}$ at $\lambda=8 \AA$ ) in the computation it had to be set to $44.2 \mathrm{~A}$ instead of the tuned value of $47.3 \mathrm{~A}$ in the experiment. An explanation probably is that this difference depends on the detection area specified for the computational optimization, which yields values between $41 \mathrm{~A}$ for a detection radius of $2 \mathrm{~cm}$, focusing on best phase map flatness at the center, and $46 \mathrm{~A}$ for a radius of $11 \mathrm{~cm}$, optimizing the average overall flatness. There is also some correlation between currents in the two correction coils of each arm.

Similar mapping of the other correction coils would require selection of smaller zones/patches/pixels of each of these coils by narrow apertures positioned in front of different zones of the coils and run an echo determination for each setting. This is time consuming due to the lowered intensity and the large number of scans that have to be performed. It has not yet been tried because of lack of available beam time.

\section{Resolution values $\mathcal{R}$}

Assuming for simplicity a Gaussian distribution of the deviations of integrals over paths $\mathcal{P}$ from the nominal path integral $\Delta J_{\mathcal{P}}=$ $J_{\mathcal{P}}-J_{0}$, a limiting form of the resolution function may approximately be written ${ }^{8}$ as

$$
\mathcal{R} \simeq \exp \left[-\left(\frac{\Sigma t}{\lambda^{2}}\right)^{2}\right],
$$

where $\Sigma$ is a measure for the relative field integral homogeneity. Considering the path ensemble $\mathcal{P}$ from start to end of the spinecho precession ranges yields the inhomogeneity measure $\delta=$ $\left[\left\langle\left(\Delta J_{\mathcal{P}} / J_{0}\right)^{2}\right\rangle_{\mathcal{P}}\right]^{1 / 2}$. The parameter $\Sigma=2 \pi\left(h / m_{n}\right) \delta$ of Eq. (4) is proportional to $\delta$, i.e., $\Sigma \simeq \delta \times 2.5 \times 10^{5} \AA^{2} / \mathrm{ns}$.

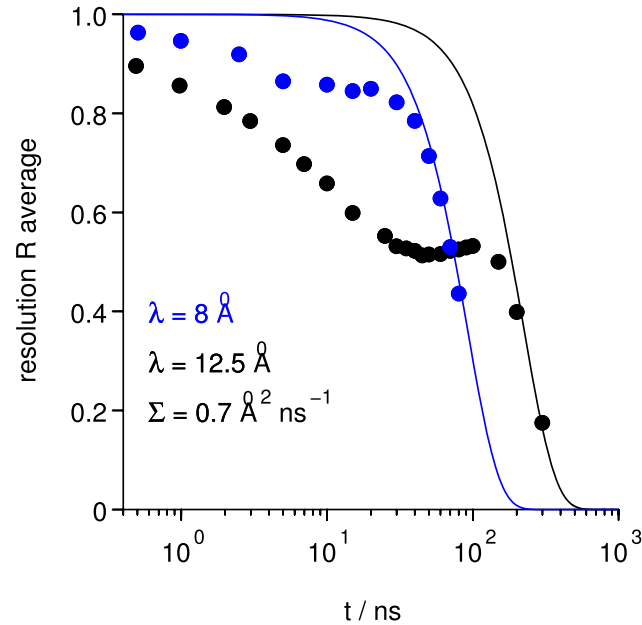

FIG. 16. Measured average resolution (weighted average over the detector area using $1 \times 1 \mathrm{~cm}^{2}$ pixels). Lines: $\exp \left[-\left(\Sigma t / \lambda^{2}\right)^{2}\right]$.

Figures 16 and 17 show the observed resolution values as obtained by using the primary data from $1 \times 1 \mathrm{~cm}^{2}$ pixel evaluation as significance weighted average over the whole illuminated detector area (Fig. 16) and the central spot (Fig. 17) at Q-values of $0.1 \AA^{-1}$ for $\lambda=8 \AA$ and $0.03 \AA^{-1}$ for $\lambda=12.5 \AA$. Whether discrepancies between the measured resolution and the simple estimate from Eq. (4) at short and intermediate times are due to depolarization effects or other dephasing influences is not yet clear. In order to understand them, further investigations are planned.

The comparison with Eq. (4) in the large Fourier time regime suggests total inhomogeneities $(\delta)$ of about $2.5 \mathrm{ppm}$ for the center and $3.5 \mathrm{ppm}$ for the large area, close to the expectation from computation.

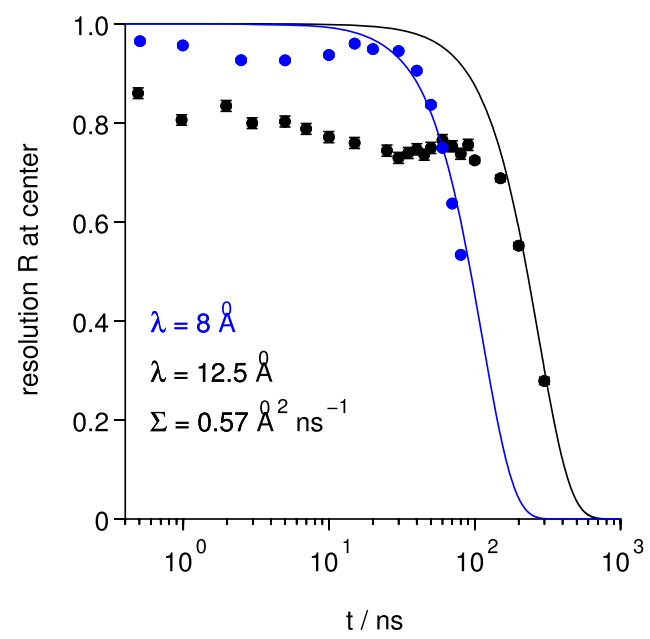

FIG. 17. Measured average resolution, average over the central spot $(9-15$ $1 \times 1 \mathrm{~cm}^{2}$ pixels). Lines: $\exp \left[-\left(\Sigma t / \lambda^{2}\right)^{2}\right]$. 
TABLE IV. Resolution at large scattering angles with correction element settings as for low $Q$ without further readjustment! The wavelength was $\lambda=8 \AA$.

\begin{tabular}{lccccc}
\hline \hline $2 \theta(\mathrm{deg})$ & $Q\left(\AA^{-1}\right)$ & $\tau(\mathrm{ns})$ & Pixel size & Active area $\left(\mathrm{cm}^{2}\right)$ & $\mathcal{R}_{\text {average }}$ \\
\hline 45 & 0.6 & 30 & $2 \times 2$ & 720 & 0.81 \\
45 & 0.6 & 80 & $2 \times 2$ & 780 & 0.49 \\
70 & 0.9 & 30 & $2 \times 2$ & 710 & 0.63 \\
70 & 0.9 & 70 & $2 \times 2$ & 700 & 0.34 \\
\hline \hline
\end{tabular}

\section{Large scattering angles}

The efficient fringe field compensation, in particular along the coil axis, allows for high resolution at large scattering angle with little or even no further readjustment of corrections. In the up to now available beam time, just the latter (no readjustment) had been tried and used for a first experiment at $Q$-values up to $0.9 \AA^{-1}$ using a wavelength of $8 \AA$. The observed average resolution values are given in Table IV. The average is over the useful detector area, which is about $70 \%$ of the total detector; the resolution at the center is still well above the quoted average values. We expect that these values can be improved further by slightly readjusting the correction coil positions at the sample sides or using the current sheets as shifters. However, due to the low intensity of even the best scattering TiZr resolution sample, in the moment, there was no beam time left to investigate this in detail.

\section{E. Phase control}

The most sensitive property of the NSE spectrometer, which is most prone to influence from magnetic fluctuations, is the field integral symmetry, often expressed in terms of the symmetry phase. Predictability and reproducibility of phases is very important for an unconditional validity of the ultimate values of the intermediate scattering function that is extracted from a set of experiments comprising resolution, sample, and background. The resolution runs are to be used to determine the values of the symmetry phases. This requires sufficient statistics and echo amplitude. For the sample runs, it may be still feasible to fix the phase using the actual data, provided that the intensity (statistics) and the echo amplitude are sufficient. For a background run in general, both conditions are at best only poorly met. Thus, fixing the phase by the resolution runs would be the canonical procedure during evaluation. In fact this is the best option at the SNS-NSE spectrometer where all external influences are marginalized by a magnetic shielding.

Unfortunately the situation is worse for all other NSE instruments without shielding. There, typical phase drifts between resolution and sample experiments (which may be days or weeks apart) are observed. Drifts may occur due to drifts in spectrometer components because of thermal drift or changes in the environment, e.g., crane motion, magnets operated by other instruments, and moving of larger ferromagnetic structures. The latter might be mitigated by a magnetic shielding if it can be afforded in terms of space and cost. To cope with any kinds of such drifts, the phase information is updated by analyzing the sample data as much as possible, a procedure that trades an increase in statistical noise with a reduction in systematical errors. It will fail for low residual echo amplitudes and/or weakly scattering samples. For the background, one has no choice than to resort to the previously determined phases.

Thus if no magnetic shielding is possible, monitoring and modeling the external influences is the only resort. The stability of the new super-J-NSE-due to the monolithic superconducting coil setis such that an approach to compute phases by taking into account all known, sufficiently weak, and distant external influences seems to be a realistic option.

So far for a proper prediction of the required zero-phase currents by the current setting, the algorithm showed the necessity to roughly account for the gradients of the background fields in the guide hall by including a virtual coil and the contribution of half a turn of the $\mathrm{ccl}$ correction coil current (to account for the current feed).

\section{CONCLUSION}

Figure 18 is a quick overview of the possibility offered by the new J-NSE in terms of feasible Fourier times, as a function of the current in the main precession coils. The curves are drawn from experimental data. The velocity selector of the instrument allows us to use wavelengths larger than $4.5 \AA$ with a gap interval $13 \AA<\lambda$ $<16 \AA$ due to mechanical resonances. So far the instrument has been optimized and tested to work between $6 \AA$ and $13 \AA$. The maximum main current value of $140 \mathrm{~A}$ in Fig. 18 is not yet determined by the superconducting system $\left(I_{\max }=220 \mathrm{~A}\right)$, but it is rather a limit imposed by current technical constraints on other power supplies and auxiliary coils.

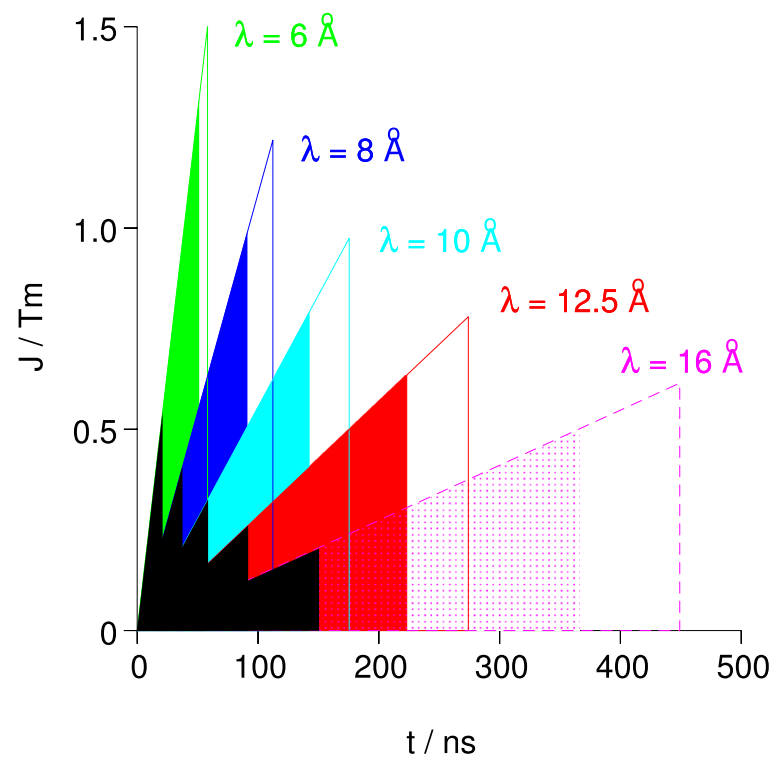

FIG. 18. Experiment-verified ranges of feasible field-integrals vs Fourier times for typical wavelengths, in the limit $\mathcal{R} \geq 1 / e$. For each wavelengths, three regions have been identified: The Fourier time delimited by the solid line is for a resolution up to the 1/e criterion applied to the central part resolution values from Fig. 17; the Fourier time delimited by the solid, colored region is for the same limit for $\mathcal{R}$ but integrated over $70 \%$ of the detector area; the solid, black regions represent the performance of the old J-NSE. Only the performance for $\lambda=16 \AA$ is extrapolated. 
For practical use, a $1 / e$-limit for $\mathcal{R}_{\text {average }}$ is a reasonable default. Under this condition, the new J-NSE Phoenix reaches now 220 ns at $\lambda=12.5 \AA$, while with the previous version of J-NSE with the original copper coils, it proved very difficult to exceed $90 \mathrm{~ns}\left(\mathcal{R}_{\mathrm{av}, \text { old }} \simeq 0.399\right.$ average over the useable $68 \%$ of the detector area). Currently the correction and adjustment state of the new spectrometer is such that for $350 \mathrm{~ns}$ at $\lambda=12.5 \AA$, the average resolution is $\mathcal{R}_{\text {average }}=0.22 \approx 0.6 / \mathrm{e}$. The necessary wavelength for a given maximum Fourier time is reduced by $\sqrt{2.5}$, e.g., experiments that used to require $\lambda=12.5 \AA$ now can be done with $\lambda=8 \AA$ with considerably enhanced neutron flux. The differential neutron flux at the long wavelength side of the cold thermal Maxwell spectrum is $d \Phi(\lambda) / d \lambda \propto \lambda^{-5}$; however, because of the use of a velocity selector, the fixed $\Delta \lambda / \lambda$ width leads to a flux factor at the sample $\propto \lambda^{-4}$; together with the increasing extinction of long wavelength neutrons by windows, in-beam elements, and air gaps, the intensity scales as $\lambda^{-4} \times \exp \left(-\Sigma_{\mathrm{a}} \lambda\right)$, with $\Sigma_{\mathrm{a}}$ being the total absorption/extinction coefficient with $1 / v$-characteristics. For a typical situation, this combination will amount to a factor of 8-10 for the above considered wavelength ratio.

Besides the primary resolution enhancement due to the optimized coil geometry, the fringe field compensation allows easy access to large scattering angles at full resolution. Furthermore, the compensation together with the inherent lack of thermal expansion effects of the cold superconducting coil set leads to inherent phase (i.e., symmetry) stability independent of residual flipper position drifts in the millimeter-range.

Even with the still limited operation experience due to restricted availability of neutrons since the start of full operation of the J-NSE Phoenix spectrometer, we can already say that the expected resolution improvement of 2.5 has been fully achieved and that the measuring efficiency gain is nearly one order of magnitude.

\section{SUPPLEMENTARY MATERIAL}

See supplementary material for more details on the optimal setting of the correction coils and further information about the short-Fourier-time setup.

\section{ACKNOWLEDGMENTS}

The coil design is based on results obtained in the BMBF ESS Design-Update Project No. 05E10CJ1.
We further acknowledge the essential technical support by Ch. Tiemann, R. Achten, U. Giesen, and H. Soltner, FZJ, ZEA-1 (construction and correction coils); F. Beule, G. Vehres, and F. Suxdorf, FZJ, JCNS-1 (electronics and interface programming); and M. Bednarek, FZJ, G-ELI, and S. Staringer, V. Ossovyi, A. Nebel, and G. Brandl, Ch. Felder FZJ, JCNS-FRM-II (installation and operation).

\section{REFERENCES}

${ }^{1}$ O. Holderer, M. Monkenbusch, R. Schaetzler, H. Kleines, W. Westerhausen, and D. Richter, in 4th European Conference on Neutron Scattering, Lund, Sweden, June, 2007, Meas. Sci. Technol. 19, 034022 (2008).

${ }^{2}$ S. Pasini and M. Monkenbusch, Meas. Sci. Technol. 26, 035501 (2015)

${ }^{3}$ S. Pasini, M. Monkenbusch, and T. Kozielewski, in VI European Conference On Neutron Scattering (ECNS), Univ Zaragoza, CSIC, Mat Sci Inst Aragon, Zaragoza, SPAIN, August 30-September 04, 2015, J. Phys.: Conf. Ser. 746, 012006 (2016).

${ }^{4}$ F. Mezei, Nucl. Instrum. Methods 164, 153 (1979).

${ }^{5}$ C. Zeyen and P. Rem, Meas. Sci. Technol. 7, 782 (1996).

${ }^{6}$ T. Takeda, S. Komura, H. Seto, M. Nagai, H. Kobayashi, E. Yokoi, C. Zeyen, T. Ebisawa, S. Tasaki, Y. Ito, S. Takahashi, and H. Yoshizawa, Nucl. Instrum. Methods Phys. Res., Sect. A 364, 186 (1995).

${ }^{7}$ B. Farago, ILL-Grenoble, private communication (2018).

${ }^{8}$ M. Ohl, M. Monkenbusch, N. Arend, T. Kozielewski, G. Vehres, C. Tiemann, M. Butzek, H. Soltner, U. Giesen, R. Achten, H. Stelzer, B. Lindenau, A. Budwig, H. Kleines, M. Drochner, P. Kaemmerling, M. Wagener, R. Moeller, E. B. Iverson, M. Sharp, and D. Richter, Nucl. Instrum. Methods Phys. Res., Sect. A 696, 85 (2012).

${ }^{9}$ M. Monkenbusch, R. Schatzler, and D. Richter, Nucl. Instrum. Methods Phys. Res., Sect. A 399, 301 (1997).

${ }^{10}$ CAENELS Easy-Driver and CAENELS Fast-PS-M and Fast-PS-1K5 series, CAENels, Trieste, Italy.

${ }^{11}$ The superconducting coils systems were obtained from BABCOCK NOELL GmbH, 97080 Würzburg, Germany.

${ }^{12}$ The residual hysteresis may be erased by raising the temperature above the critical temperature of the NbTi wire $(9 \mathrm{~K})$. This was experienced once with the SNS-NSE superconducting coils as a compressor failure occurred for the cooling of one coil only. Continuation of the measurement revealed a phase jump of about $60^{\circ}$ upon implicit introduction of an asymmetry. After completing the current cycle, the asymmetry was again removed.

${ }^{13}$ B. Farago, M. Monkenbusch, K. Goecking, D. Richter, and J. Huang, Physica B 213, 712 (1995).

${ }^{14}$ M. Monkenbusch, Nucl. Instrum. Methods Phys. Res., Sect. A 287, 465 (1990).

${ }^{15}$ PI type N-216K027, Physics Intruments, 76228 Karlsruhe, Germany.

${ }^{16}$ Construction by ZEA, FZJ incorporating precision amagnetic rails from Feinmechanik Ulrich Klein GmbH, 42551 Velbert, Germany. 